\title{
COMMUNICATION
}

\section{Cardiac tamponade due to Actinomyces odontolyticus infection of a dentigerous cyst}

\section{Broly E DDS1 ${ }^{1}$, Risse J MD2 ${ }^{2}$, Maschino F DDS1 ${ }^{1}$, Wahl D MD PhD2 ${ }^{2}$}

1 - Nancy University Hospital, Department of Oral Pathology, Oral Medicine and Oral Surgery, Odontology faculty,

Introduction

The dentigerous or marginal posterior cyst is the most common of non-inflammatory paradental odontogenic cysts.

Actinomyces odontolyticus (Ao) is a non-spore-forming gram-positive facultative anaerobic bacteria originally known as a commensal bacterium of the oral cavity ${ }^{2}$.

We report the first case of acute pericarditis with the same strain of $A 0$ as an otherwise asymptomatic dentigerous cyst.

\section{Case report}

A 52-year-old woman was referred to the dental surgery department, in the Oral Pathology and Oral Surgery unit to find a causal dental lesion after discovery of $A o$ in pericardial fluid.

Bacteriological workout of pericardial fluid was positive for Ao. The $3^{\text {rd }}$ left mandibular molar (38) presented on its distal side a radio-brightness in a curving bow shape compatible with the image of a dentigerous cyst. We decided to realize a bacteriological sampling by puncture of the cyst then to realize the avulsion of 38 and the enucleation of the cyst. The sample confirmed the presence of the same strain of Ao than found in pericardial fluid.

The patient has a favourable clinical outcome after glucocorticoids cessation and antibiotic treatment.

\section{Discussion}

Human actinomycosis is an infection caused mainly by six species of Actinomyces, a gram positive anaerobic bacteria, usually colonizing ENT region, colon and urogenital tract ${ }^{6}$. Clinical examination is insidious without any specific symptoms. Cardiac involvment is exceptional ${ }^{7}$.

Ao is a bacterium across the commensal oral flora and is included in the pink complex defined by Socransky et al. ${ }^{10}$. The particularity of this case holds in two points; the first one is an acute pericarditis due to Ao of dental origin in a patient immunocompromised by glucocorticoids. The second is the particularity of the primary infection site, without any particularity except this dentigerous cyst, latent and asymptomatic since the eruption of this tooth. Due to its variety of 
clinical presentations and its capacity to mime numerous pathologies, we insist through this case on the importance of an odontological check-up in case of a cardiac wall damage in an infectious context.

\section{Conclusion}

Through this rare case of cardiac tamponade due to Ao we emphasize the importance of careful dental examination in case of an underlying infection. A dental and maxillary sinus clinical and radiological assessment should be the norm and any caries or periodontal damage should be treated promptly. Routine bacterial sampling must be be carried out during dental assessment.

\section{References}

1 - Anderson DW and al.: Dentigerous cyst of mandible presenting as sepsis. AM J Emerg Med, 2014 32:1561.e3-4.

2 - Batty I: Actinomyces odontolyticus, a new species of actinomycete regularly isolated from deep carious dentine. J Path Bactiol, 1958, 75:455-9.

7 - Fife TD et al.: Pericardial actinomycosis: case report and review. Rev Infect Dis, 1991, 13:120-6. 